\title{
Trichostatin A-induced Apoptosis is Mediated by Krüppel-like Factor 4 in Ovarian and Lung Cancer
}

\author{
Sadeghi Zohre ${ }^{1,5}$, Nejati-Koshki Kazem ${ }^{5}$,Akbarzadeh Abolfazl' ${ }^{2}$, Rahmati-Yamchi \\ Mohammad $^{1,3}$, Movassaghpour Aliakbar ${ }^{4}$, Alizadeh Effat ${ }^{5}$, Davoudi Zahra ${ }^{5}$, \\ Dariushnejad Hassan ${ }^{5}$, Zarghami Nosratollah ${ }^{1,3,5 *}$
}

\begin{abstract}
Background: The istone deacetylase (HDAC) inhibitor trichostatin A (TSA) is known to mediate the regulation of gene expression and antiproliferation activity in cancer cells. Krüppel-like factor 4 (klf4) is a zinc fingercontaining transcription factor of the SP/KLF family, that is expressed in a variety of tissues and regulates cell proliferation, differentiation, tumorigenesis, and apoptosis. It may either either function as a tumor suppressor or an oncogene depending on genetic context of tumors. $\underline{\text { Aims: }}$ : In this study, we tested the possibility that TSA may increase klf4 expression and cancer cell growth inhibition and apoptosis in SKOV-3 and A549 cells. Materials and Methods: The cytotoxicity of TSA was determined using the MTT assay test, while klf4 gene expression was assessed by real time PCR andto ability of TSA to induce apoptosis using a Vybrant Apoptosis Assay kit. Results: Our results showed that TSA exerted dose and time dependent cytotoxicity effect on SKOV-3 and A549 cells. Moreover TSA up-regulated klf4 expression. Flow cytometric analysis demonstrated that apoptosis was increased after TSA treatment. Conclusions: Taken together, this study showed that TSA increased kIf4 expression in SKOV3 and A549 cell lines, consequently, klf4 may played a tumor-suppressor role by increasing both cell growth inhibition and apoptosis. This study sheds light on the details of molecular mechanisms of HDACI-induced cell cycle arrest and apoptosis.
\end{abstract}

Keywords: Histone deacetylase inhibitor - Trichostatin A - klf4 - apoptosis

Asian Pac J Cancer Prev, 15 (16), 6581-6586

\section{Introduction}

Histone deacetylase inhibitors (HDACi) have emerged as a new class of targeted anticancer agents which mediates the regulation of gene expression and induces growth inhibition, apoptosis, differentiation and angiogenesis in a various of tumor cell lines (Glaser, 2007; Platta et al., 2007; Rasheed et al., 2007; Wu et al., 2007; Sonnemann et al., 2008; Bellarosa et al., 2012; Francisco et al., 2012; Shan et al., 2012; Feng et al., 2013). Owing to the high activity of HDACs in some types of cancers and reversible histone acetylation activity, HDAC has been recognized as excellent drug target for cancer therapy( Wdcae, 2000; Weichert et al., 2008).

There are several types of HDACi, including SAHA, butyrate and trichostatin A (TSA). Hydroxamic acid HDAC inhibitor TSA is a compound with antifungal activity that was initially isolated from Streptomyces hygroscopicus (Owen et al., 1998). TSA is a specific and strong HDA-inhibitor (HDACi) which can alter gene expression and induce apoptosis in a variety of cancer cells at very low concentration. The HDACi have been documented to increase the expression of pro-apoptotic proteins and caspase and repress the expression of antiapoptotic Proteins (Sawa et al., 2001; Chen et al., 2002; Liu et al., 2006; Wilson et al., 2011; Horing et al., 2013; Zhang et al., 2013; Peiffer et al., 2014). The activity of a wide variety of non-histonin transcription factors altered by acetylation, and affected by HDACi (Van et al., 1996; Glaser et al., 2003).

Several transcription factors such as NF-kB, P53, E2F, Myc and klf4 are altered in human cancers (Mees et al., 2009). Kruppel-like factor 4 (klf4), formerly identified as a gut enriched KLF (GKLF) or epithelial zinc finger(EZF), is a zinc finger-containing transcription factor of the SP/ KLF family, that expressed in a variety of tissues and regulates a range of biological processes, including cell proliferation, differentiation, tumorigenesis, apoptosis , and embryogenesis (Shields et al., 1996; Rowland et al., 2005; Wei et al., 2005; Wang et al., 2010; Aksoy et al., 2014). As a TF klf4 activates and represses the transcription of several genes that are involved in cell-cycle regulation

${ }^{1}$ Hematology and Oncology Research Center, ${ }^{2}$ Department of Medical Nanotechnology, ${ }^{5}$ Department of Medical Biotechnology, Faculty of Advanced Medical Science, ${ }^{3}$ Department of Clinical Biochemistry, ${ }^{4}$ Department of Hematology, Faculty of Medicine, Tabriz University of Medical Sciences, Tabriz, Iran*For correspondence: zarghami@tbzmed.ac.ir 
and proliferation depending on the cellular context (Chen et al., 2003). klf4 activates the promoters of the negative cell-cycle-regulatory cyclin-dependent kinase inhibitor p21WAF1/Cip1 and p27Kip1 genes (Zhang et al., 2000; Daoyan et al., 2008). The studies Described that klf4 and p53 synergistically activate the p21WAF1/Cip1 promoter and are bound concurrently to the promoter of p21WAF1/ Cip1 (Zhang et al., 2000). In addition, klf4 represses the promoters of several cell cycle-promoting genes such as CCND1 (cyclin D1)and CCNB1 (cyclin B1) (Shie et al., 2000; Yoon and Yang, 2004). These lead to cell cycle arrest at the G1/S phase and is also necessary to prevent the G2/M progression after DNA damage (Yoon et al., 2003; Yoon and Yang, 2004). As previously discussed, klf4 implicated in the regulation of apoptosis(Chen et al., 2000; hnishi et al., 2003). klf4 binds to the promoter of survivin gene, which is necessary for caspase inactivation and repress this promoter (Glaser et al., 2003).

Several lines of evidence indicated that klf4 either function as a tumor suppressor or an oncogen depending on genetic context of tumors. klf4 was primary recognized as a tumor suppressor in a variety of malignancies such as gastric, colon, ovarian, and lung cancers and its inactivation may play an essential role in tumor progression. Following studies have suggested an oncogenic role for klf4 in other cancers, including breast and dysplastic oral squamous epithelium (Rowland et al., 2005; Yori et al., 2011; Yu et al., 2011).

Up to our knowledge, the effect of TSA on klf4 expression was not studied previously. Also, regarding the importance of TFs in control of cancer progression, druginduced apoptosis, and cell fate, our study was directed for elucidating the effect of HDACi TSA on proliferation and apoptosis of ovarian and lung cells. In addition we examined the possibility that klf4 may be a target gene for HDACi TSA and probable involvement of it in the inhibition of tumor proliferation. The findings of current paper may help elucidating mechanism of action of TSA in ovarian and lung cancer cell lines.

\section{Materials and Methods}

\section{Cell culture}

Ovarian and Lung cancer epithelial-like cell line, SKOV-3 and A549,were obtained from Pasteur Institute cell bank of Iran (code: C209,C137). These cells were cultured in RPMI 1640 medium(Gibco, Invitrogen, UK) supplemented with $10 \%$ fetal bovine serum (FBS (Gibco,Invitrogen, UK)) ,100 $\mu \mathrm{g} / \mathrm{mL}$ streptomycin (Merck co, Germany), and $100 \mathrm{U} / \mathrm{ml}$ penicillin (Serva co,Germany), and $0.37 \%$ (w/v) $\mathrm{NaHCO}_{3}$. Cells were incubated at $37^{\circ} \mathrm{C}$ in $5 \% \mathrm{CO}_{2}$ and $95 \%$ humidified air. Media were changed twic weekly (45-64).

In vitro cytotoxicity

The cytotoxicity of TSA (Sigma-Aldrich) was determined using the MTT (3- (4,5- dimethyl-2-yl)-2, 5-diphenyl tetrazolium bromide) assay (MTT;SigmaAldrich), which is based on the conversion of the yellow tetrazolium salt MTT to purple formazan crystals by mitochondrial dehydrogenases. In brief, Viable cells were counted using trypan blue $(0.02 \%)$ and seeded in 96-well
Table 1. Sequences of Primers for RT-PCR

\begin{tabular}{lccc}
\hline Primer name & $\begin{array}{c}\text { Primer } \\
\text { length }\end{array}$ & $\begin{array}{c}\text { Sequence } \\
\text { (5' to 3') }\end{array}$ & $\begin{array}{c}\text { Product } \\
\text { size (bp) }\end{array}$ \\
\hline klf4 forward & 20 & GGCACTACCGTAAACACACG & 40 \\
klf4 reverse & 20 & CTGGCAGTGTGGGTCATATC & 40 \\
$\beta$-actin forward & 20 & TCCCTGGAGAAGAGCTACG & 131 \\
$\beta$-actin reverse & 20 & GTAGTTTCGTGGATGCCACA & 131 \\
\hline
\end{tabular}

plates at a density of $3 \times 10^{3}(\mathrm{SKOV}-3)$ and $2 \times 10^{2}$ (A549) cells/well and allowed to attach and equilibrate for $24 \mathrm{~h}$ in a humidified atmosphere of $95 \%$ air and $5 \% \mathrm{CO}^{2}$. They then treated with various concentrations of TSA for 24,48 and $72 \mathrm{~h}$. After the end of incubation, $10 \mu \mathrm{l} \mathrm{MTT}(5 \mathrm{mg} /$ $\mathrm{ml}$ PBS) was added to each well for $4 \mathrm{~h}$ the supernatant was removed and $200 \mu$ l dimethyl sulfoxide(DMSO) were added to dissolve the formazan salt formed. Absorbance was measured at $570 \mathrm{~nm}$ using an ELISA Reader.

\section{RNA extraction and cDNA synthesis}

Cells were treated with TSA $(100 \mathrm{nM})$ for $72 \mathrm{~h}$. Total RNA from non-teated and TSA-treated cells was extracted using RNX-Plus solution(CinaGen,Iran) according to the manufacturer's instructions. Total RNA was quantified using a photospectrometer (NanoDrop nd$1000 \mathrm{uv} / \mathrm{vis}$, U.S.A.) and RNA integrity was confirmed by electrophoresis of samples on a $1.2 \%$ agarose gel. The cDNA was synthesized from $1 \mu \mathrm{g}$ of total RNA from each sample, and $1 \mu \mathrm{l}$ of random hexamer primers (Roche AppliedScience) was diluted to a final volume of $20 \mu \mathrm{l}$ in DEPC-treated water using a AccuPower RocketScript RT PreMix kit (BIONEER, Korea) according to the manufacturer's protocol. cDNA synthesis was performed under the following condition, anneal-ing at $15^{\circ} \mathrm{C}$ for 1 min, cDNA synthesis $50^{\circ} \mathrm{C}$ for $1 \mathrm{~h}$ and Heat inactivation $95^{\circ} \mathrm{C}$ for $5 \mathrm{~min}$. The samples can be stored at $-20^{\circ} \mathrm{C}$ until use.

\section{Quantitative Real-time PCR}

Real-time PCR was performed in a Corbett (Rortor Gene 6000) system using AccuPower $2 X$ Greenstar qPCR Master Mix (BIONEER, Korea). $\beta$-Actin was used as a housekeeping gene for standardization of klf4 mRNA expression. The primer sequences used in our study were showed in Table 1.

A total of $20 \mu \mathrm{l}$ of PCR reaction mixture was made using $2 \mu \mathrm{l}$ cDNA, $1 \mu \mathrm{l}$ forward primer, $1 \mu \mathrm{l}$ reverse primer, $10 \mu \mathrm{l}$ SYBR premix EX Taq, and $6 \mu$ l of DEPC-treated water. The thermal profile for real-time PCR was $95^{\circ} \mathrm{C}$, 10 minutes, 1 cycle (Holding step); $95^{\circ} \mathrm{C}, 15$ seconds, $58^{\circ} \mathrm{C} 45$ second 40 cycles (cycling step); $72-95^{\circ} \mathrm{C}, 1$ cycle (Melting step). Expression $\mathrm{f}$ each amplicon was analyzed by using the $2^{-\Delta \Delta C t}$ method, where ${ }^{\Delta \Delta C t}$ is the $\Delta \mathrm{Ct}$ (klf4) $-\mathrm{DCt}$ (actin) and $\mathrm{Ct}$ is the cycle at which the threshold is crossed. Flow cytometry analysis for apoptosis

The ability of TSA to induce apoptosis of SKOV3 and A549 cells was evaluated using the Vybrant Apoptosis Assay kit ((Invitrogen,USA), according to the manufacturer's Protocol. This assay was based on the differential permeability of the apoptotic to the green fluorescent dye (YO-PRO-1) and necrotic cells to the red fluorescent dye [propidium iodide (PI)]. YO-PRO-1 can 
enter the apoptotic cells and bind to DNA or RNA, which significantly enhances its fluorescence intensity. cells were cultured in 6 well plates for $72 \mathrm{~h}$ and then treated with various concentration of TSA. After $72 \mathrm{~h}$ treatment, Approximately $5 \times 10^{5}$ Cells collected by trypsinization and washed and re-suspended in $1 \mathrm{ml}$ of phosphatebuffered saline(PBS). YO-PRO-1 and PI was added at a final concentration of each prob $1 \mu$ then incubated for $30 \mathrm{~min}$ in the dark at $4^{\circ} \mathrm{C}$. Cells were analyzed by FACS Calibur flow cytometer (BD Biosciences, Franklin Lakes, NJ,USA) with flowjo software and the percentages of apoptotic (YO-PRO-1+/PI and necrosis (YO-PRO-1/PI+) cells were determined.

\section{Statistical analysis}

Results are presented as mean and standard error of the mean (SEM). Data were analyzed using ANOVA and Tukey's test to calculate the significance values; a p-value $<0.05$ was considered as significant. All experiments were replicated at least three times to confirm the result.

\section{Results}

Inhibitory effects of TSA on SKOV-3 and A549 cells proliferation

The MTT assay was used to evaluate viability of SKOV-3 and A549 cells exposed to different concentrations of TSA for 24, 48 and $72 \mathrm{~h}$. The result in SKOV-3 and A549 cells showed that the survival rate of treated cells decreased with the increasing concentration of TSA (see Figure 1), which was also dependent on the time of incubation. The $\mathrm{IC}_{50}$ parameters determined for TSA were 5.6, 0.7, 0.32 $\mu \mathrm{M}$ in SKOV-3 cells and 3.2,0.28, $0.06 \mu \mathrm{M}$ in A549 cells after 24, 48, and 72h treatments, respectively.

TSA Stimulates klf4 Expression in SKOV-3 and A549 cells

To examine TSA effects in klf4 mRNA expression in human Ovarian, Lung cell lines, we analyzed the level of klf4 mRNA by real time RT-PCR in SKOV-3 and A549 cell lines in the presence and absence of TSA. Changes in klf4 mRNA expression levels between the treated and Control cells were normalized to beta-actin mRNA levels and calculated by the $2^{-\Delta \Delta \text { ct }}$ formula. The results demonstrated that treatment with TSA induced an increase in klf4 mRNA levels in these cell lines. The induction of klf4 mRNA expression by TSA was reverse dose dependent. A 9.3and 15.5-fold increase occurred in SKOV-3 cells treated with 0.32 and $0.22 \mu \mathrm{M}$ TSA and 2.1 and 8.57 fold increase occurred in A549 cells treated with 0.06 and 0.04 TSA concentration.

Induction of apoptosis by TSA in SKOV-3 and A549 cells Apoptosis in SKOV-3 and A549 cells was assessed by flow cytometry. Flow cytometry was used to distinguish the uptake of YO-PRO-1 by apoptotic cells.YO-PRO-1 is a green fluorescent dye, which demonstrate considerable fluorescence enhancements upon binding to nucleic acids. While propidium iodide (PI), red fluorescence dye, is applied to stain necrotic cells. As shown in Figure 2 and 3 , in SKOV-3 cells the percentage of apoptotic cells in $0.42,0.32$, and $0.22 \mu \mathrm{M}$ concentration was $8.58,15.3$, and $19.5 \%$ and in A549 cells in 0.08, 0.06, and $0.04 \mu \mathrm{M}$ concentration was $14,18.7$, and $25.8 \%$, respectively after $72 \mathrm{~h}$ of incubation. We used different concentration of TSA according to the results of MTT assay.The concentration of TSA are around $\mathrm{IC}_{50}$ ranges. According to the results the number of apoptotic cells labeled with YO-PRO-1
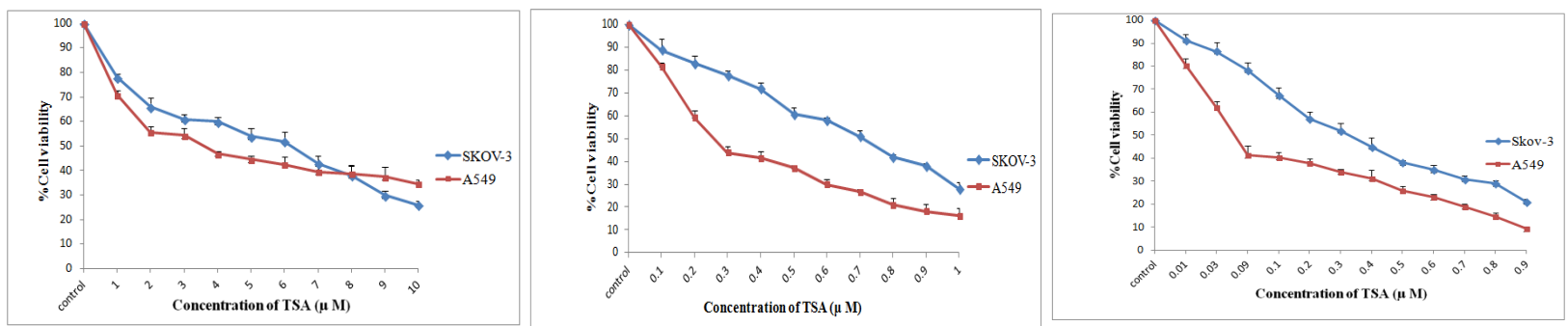

Figure 1. A: Effect of TSA on Survival of Skov-3 and A549 Cells. Cells were treated with different concentrations of TSA for $24(\mathrm{~A}), 48(\mathrm{~B})$, and $72(\mathrm{C}) \mathrm{h}$. Cell viability was measured by MTT assay
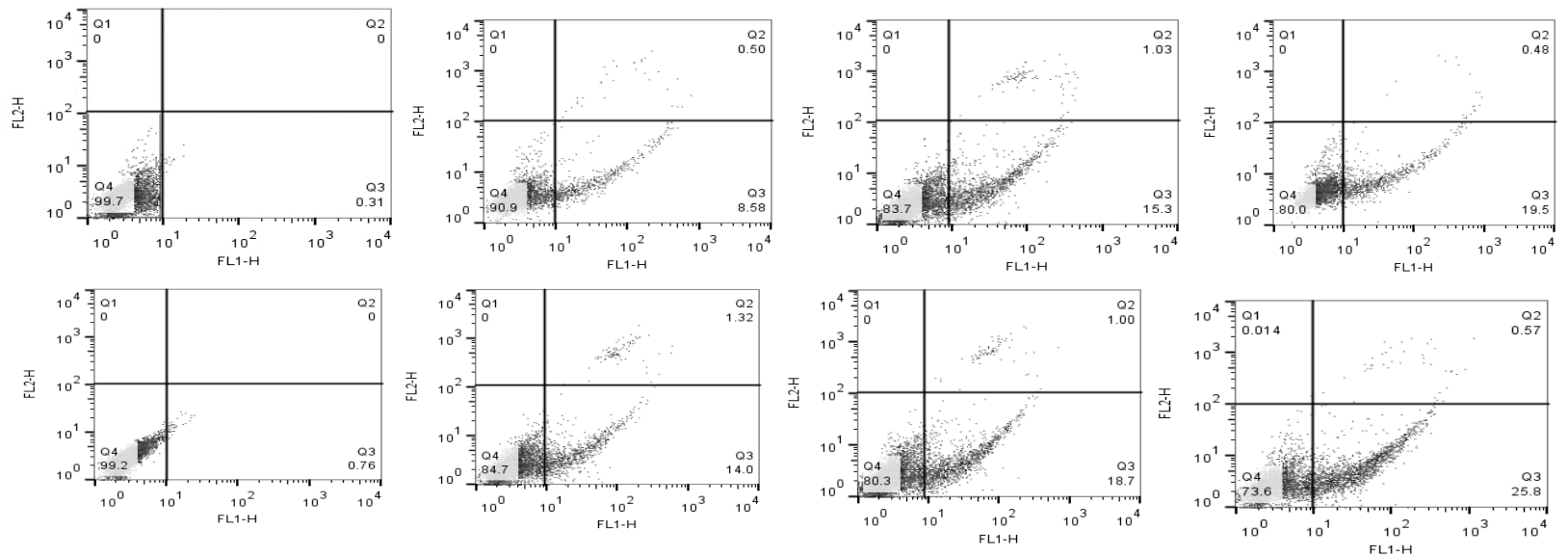

Figure 2. Apoptosis in (a) Skov-3 and (b) A549 Cells was Assessed by Flow Cytometry 


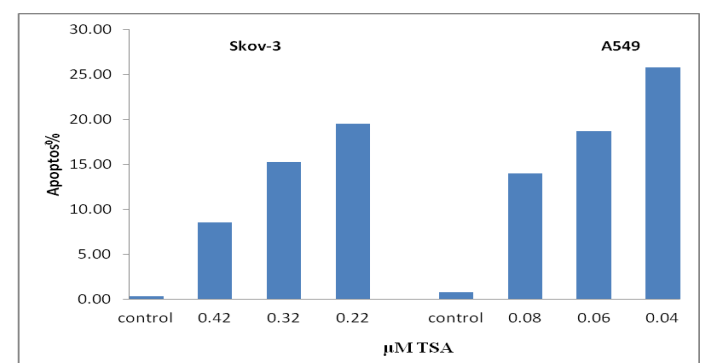

Figure 3. Effect of TSA on Apoptosis of Skov-3 and

A549 Cells. After cultured with various concentrations of TSA for $72 \mathrm{~h}$, cells were stained with yo-pro/propidium iodide, and analyzed by flow cytometry

increased with decreasing TSA concentration.

\section{Discussion}

The aim of this study was to determine the response of the cell lines: SKOV-3 and A549 from Ovarian and Lung cancers, respectively to treatment with the histone deacetylase inhibitor TSA. Previous studies have shown that Histone deacetylase inhibitors have antitumor activity in a variety of cancers and compared with conventional chemotherapeutic drugs, Histone deacetylase inhibitors have tumor selectivity and less toxicity in normal cells (Butler et al., 2000; Butler et al., 2001; Vigushin et al., 2001; Cheng et al., 2012; Zhang et al., 2012; Wang et al., 2013). Recent studies have demonstrated that TSA induces cell growth inhibition, cell-cycle arrest and apoptosis in hela, bladder, pancreatic, Glioblastoma, breast, and colon cancer cell lines through $\mathrm{Zn}$ ion chelating (Paul et al., 2000; Sawa et al., 2001; Vigushin et al., 2001; Paolo et al., 2006; Horing et al., 2013; You and Park, 2013). Epigenetical interference of HDACis is now thought to play important roles to avert onset and progression of cancer in several tumor types.

There are only a few studies examining TSA effects in human ovarian and lung cancer cell lines. We investigated the effect of TSA on the cell viability and apoptosis of human epithelial ovarian and lung carcinoma cell lines (SKOV-3 and A549 cells). Our current findings indicate that the induction of cell death by TSA was dose and time dependent and occurred at a very low concentration. In accordance with this study Guang et al showed that TSA have cytotoxicity effect in dose dependent manner in breast cancer cells and the nanomolar concentrations of TSA are effective (Guang et al., 2012).

This study showed that TSA could induce apoptosis in both cell lines SKOV-3 and A549, but the rate of apoptosis in A549 cells was more than SKOV-3. The possible reason is type of P53 gene, mutant in SKOV-3 and wild type in A549. In accordance with this data,Z.Song et al indicated that TSA induced apoptosis $17.6 \%$ after $24 \mathrm{~h}$ of treatment (38). C. Herold et al illustrated time-dependent increase of apoptosis in the hepatoma cell lines (18 and 32\% for HepG2 vs 38 and 50\% for Hep1B after 24 and 72 h TSA treatment) (Herold et al., 2002).

The anti-neoplastic properties of HDAC inhibitors are mediated by their ability to induce histone hyperacetylation, resulting in the altered transcription of key genes, some of them involving in regulation of cell proliferation and

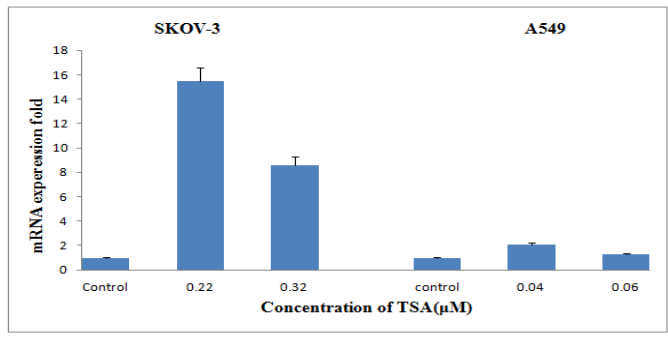

Figure 4. Effect of TSA on KLF4 Gene Expression in Skov-3 and A549 Cdells. Relative expression levels of KLF4 mRNA at various concentration points as indicated as determined by real-time PCR. The amount of KLF4 mRNA was normalized to an endogenous reference, $\beta$-actin.

apoptosis (Choudhary et al., 2009).

This study is the first to demonstrate trichostatin A effects on klf4 mRNA expression in ovarian and lung cancer cells. In present study, we exhibited TSA considerablely induced the expression of tumor suppressor gene klf4 on mRNA level in human ovarian and lung cancer cell lines. Several studies indicated that klf4 has variable effects on cell cycle arrest and induction of apoptosis depending on the genetic context of tumors (as a tumor suppressor or an oncogene). The work of Zhi Yi Chen et al that they showed TSA and butyrate upregulated klf4 mRNA levels in HT-29 cells, We achieved similar data in SKOV-3 and A549 cells. On the other hand, some papers have reaveled that klf4 mRNA expression was significantly decreased in ovarian and lung cancers (Rowland et al., 2005; Choia et al., 2006; Yori et al., 2011; Yu et al., 2011; Yoon and Roh, 2012). The down regulation of klf4 leads to uncontrolled cell proliferation and tumor development. Other reports have also shown that klf4 can repress HDAC gene expression (Ai et al., 2004). However molecular mechanisms of HDACI-induced cell cycle arrest and apoptosis, and the regulation of gene expression remain unclear and Future studies about is needed.

In conclusion, the results of the present study indicate that trichostatin A have inhibitory effect in ovarian and lung cancer cell lines(SKOV-3 and A549). This inhibition was dose and time dependent. The result of flow cytometry show trichostatin A induce apoptosis in dose dependent manner. Increased klf4 expression in SKOV3 and A549 cell lines, consequently, klf4 may played a tumor-suppressor role by increasing both the cell growth inhibition and appaptosis. Also, we emphasized the possibility of klf4 as a downstream target of TSA. This study can shed light on the details of molecular mechanisms of HDACI-induced cell cycle arrest and apoptosis.

\section{Acknowledgements}

This work was supported by Hematology and Oncology research center of Tabriz University of Medical Sciences and we thank this centre for funding the study.

\section{References}

Ai W, Liu Y, Langlois M, Wang TC (2004). Kruppel-like factor 4 (KLF4) represses histidine decarboxylase gene expression 
through an upstream Sp1 site and downstream gastrin responsive elements. J Biol Chem, 279, 8684-93.

Akbarzadeh A, Ghasemali S, Nejati K, et al (2013). Study of inhibitory effect of $\beta$-cyclodextrin-helenalin complex on HTERT gene expression in T47D breast cancer cell line by real time quantitative PCR (q-PCR). Asian Pac J Cancer Prev, 14, 6949-53.

Akbarzadeh A, Hosseininasab S, Davaran S, et al (2014). Synthesis, characterization, and In vitro studies of PLGAPEG nanoparticles for oral Insulin delivery. Chem Biol Drug Des, 3, 1-9.

Akbarzadeh A, Mikaeili H, Zarghami N, et al (2012). Preparation and in-vitro evaluation of doxorubicin-loaded $\mathrm{Fe} 3 \mathrm{O} 4$ magnetic nanoparticles modified with biocompatible copolymer. Int J Nanomedicine, 7, 1-16.

Akbarzadeh A, Nejati-Koshki K, Mahmoudi Soghrati M, et al (2013). In vitro studies of NIPAAM-MAA-VP copolymercoated magnetic nanoparticles for controlled anticancer drug release. JEAS, 3, 108-15.

Akbarzadeh A, Omidfar K, Ahmadin A, et al (2014). An electrochemical immunosensor for digoxin using core-shell gold coated magnetic nanoparticles as labels. Mol Biol Rep, 41, 1659-68.

Akbarzadeh A, Pourhassan-Moghaddam M, Rahmati-Yamchi M, et al (2013). Protein detection through different platforms of immuno-loop-mediated isothermal amplification. Nanoscale Res Lett, 8, 485-95.

Akbarzadeh A, Rezaei A, Nejati-Koshki K, et al (2014). Synthesis and physicochemical characterization of biodegradable star-shaped poly lactide-co-glycolide- $\beta$ -cyclodextrin copolymer nanoparticles containing albumin. $J$ Adv Nanoparticles, 3, 1-9.

Akbarzadeh A, Rezaei-Sadabady R, Zarghami N, et al (2013). Studies of the relationship between structure and antioxidant activity in interesting systems, including tyrosol, hydroxytyrosol derivatives indicated by quantum chemical calculations. Soft, 2, 13-8.

Akbarzadeh A, Samiei M, Davaran S, et al (2012). Magnetic nanoparticles: preparation, physical properties, and applications in biomedicine. Nanoscale Res Lett, 7, 14-26.

Akbarzadeh A, Samiei M, Joo SW, et al (2012). Synthesis, characterization and in vitro studies of doxorubicin-loaded magnetic nanoparticles grafted to smart copolymers on A549 lung cancer cell line. J Nanobiotechnol, 10, 46-52.

Akbarzadeh A, Zarghami N, Mikaeili H, et al (2012). Synthesis, characterization and in vitro evaluation of novel polymercoated magnetic nanoparticles for controlled delivery of doxorubicin. Nanotechnol Sci Appl, 5, 13-25.

Aksoy I, Giudice V, Delahaye E, et al (2014). Klf4 and Klf5 differentially inhibit mesoderm and endoderm differentiation in embryonic stem cells. Nat Commun, 5, 1-15.

Bellarosa D, Bressan A, Bigioni M, et al (2012). SAHA/ Vorinostat induces the expression of the CD137 receptor/ ligand system and enhances apoptosis mediated by soluble CD137 receptor in a human breast cancer cell line. Int $J$ Oncol, 41, 1486-94.

Butler LM, Agus DB, Scher HI, et al (2000). Suberoylanilide hydroxamic acid, an inhibitor of histone deacetylase, suppresses the growth of prostate cancer cells in vitro and in vivo. Cancer Res, 60, 165-70.

Butler LM, Webb Y, Agus DB, et al (2001). Inhibition of transformed cell growth and induction of cellular differentiation by pyroxamide, an inhibitor of histone deacetylase. Clin Cancer Res, 7, 962-70.

Chen X, Erika MW, Gao SY, Yang VW (2003). Transcriptional profiling of Krüppel-like factor 4 reveals a function in cell cycle regulation and epithelial differentiation. $\mathrm{J} \mathrm{Mol} \mathrm{Biol,}$
326, 665-77.

Chen Z, Clark S, Birkeland M, et al (2002). Induction and superinduction of growth arrest and DNA damage gene 45 (GADD45) alpha and beta messenger RNAs by histone deacetylase inhibitors trichostatin A (TSA) and butyrate in SW620 human colon carcinoma cells. Cancer Lett, 188, 127-40.

Chen ZY, Shie J, Tseng C (2000). Up-regulation of gut-enriched kruppel-like factor by interferon-gamma in human colon carcinoma cells. FEBS Lett, 477, 67-72.

Chen ZY, Rex S, Tseng CC (2004). Kruppel-like factor 4 is transactivated by butyrate in colon cancer cells. J Nutr, 134, 792-8.

Cheng DD, Yang QC, Zhang ZH, Yang CX, Liu YW (2012). Antitumor activity of histone deacetylase inhibitor trichostatin a in osteosarcoma cells. Asian Pac J Cancer Prev, 13, 1395-9.

Choi BJ, Cho YG, Song JW, et al (2006). Altered expression of the KLF4 in colorectal cancers. Pathology Res Pract, 202, 585-9.

Choudhary C, Kumer C, Gnad F, et al (2009). Lysine acetylation targets protein complexes and co-regulates major cellular functions. Science, 325, 834-40.

Cress WD, Seto E (2000). Histone Deacetylases, Transcriptional Control, and Cancer. J Cell Physiol, 184, 1-16.

Ebrahimnezhad Z, Zarghami N, Keyhani M, et al (2013). Inhibition of hTERT gene expression by silibinin-loaded PLGA-PEG-Fe3O4 in T47D breast cancer cell line. Bioimpacts, 3, 67-74.

Feng L, Pan M, Sun J, et al (2013). Histone deacetylase 3 inhibits expression of PUMA in gastric cancer cells. $\mathrm{J} \mathrm{Mol}$ Med, 91, 49-58.

Francisco R, Perez-Perarnau A, Cortes C, et al (2012). Histone deacetylase inhibition induces apoptosis and autophagy in human neuroblastoma cells. Cancer Lett, 1, 42-52.

Ghasemali S, Nejati-Koshki K, Tafsiri E, et al (2013). Inhibitory effects of $\beta$-cyclodextrin-helenalin complexes on H-TERT gene expression in the T47D breast cancer cell line - results of real time quantitative PCR. Asian Pac J Cancer Prev, 14, 6949-53.

Glaser KB, Staver MJ, Waring JF, et al (2003). Gene expression profiling of multiple histone deacetylase (HDAC) inhibitors: defining a common gene set produced by HDAC inhibition in T24 and MD acarcinoma cell lines. Mol Cancer Ther, 2, 151-63.

Glaser KB (2007). HDAC inhibitor: clinical update and mechanism-based potential. Biochem Pharmacol, 74, 659-71.

Herold C, Ganslmayer M, Ocker M, et al (2002). The histonedeacetylase inhibitor trichostatin a blocks proliferation and triggers apoptotic programs in hepatoma cells. J Hepatol, 36, 233-40.

Horing E, Podlech O, Silkenstedt B, et al (2013). The histone deacetylase inhibitor trichostatin a promotes apoptosis and antitumor immunity in glioblastoma cells. Anticancer Res, 33, 1351-60.

Liu T, Kulijaca S, Tee A, Marshall GM (2006). Histone deacetylase inhibitors: multifunctional anticancer agents. Cancer Treat Rev, 32, 157-65.

Marks PA, Richard A, Rifkind RA (2000). Histone deacetylase inhibitors: inducers of differentiation or apoptosis of transformed cells. J Nati Cancer Inst, 92, 1210-6.

Mees S, Nemunai J, Senzer N (2009). Transcription factors: their potential as targets for anindividualized therapeutic approach to cancer. Cancer Gene Ther, 16, 103-12.

Mollazade M, Nejati-Koshki K, Akbarzadeh A, et al (2013). PAMAM dendrimers augment inhibitory effects of 
curcumin on cancer cell proliferation: possible inhibition of telomerase. Asian Pac J Cancer Prev, 14, 6925-8.

Nejati-Koshki K, Zarghami N, Pourhassan-Moghaddam M, et al (2012). Inhibition of leptin gene expression and secretion by silibinin: possible role of estrogen receptors. Cytotechnology, 64, 719-26.

Nejati-Koshki K, Akbarzadeh A, Pourhasan-Moghaddam M, et al (2013). Inhibition of leptin and leptin receptor gene expression by silibinin-curcumin combination. Asian Pac J Cancer Prev, 14, 6595-9.

Nasiri M, Zarghami N, Koshki KN, et al (2013). Curcumin and silibinin inhibit telomerase expression in T47D human breast cancer cells. Asian Pac J Cancer Prev,14, 3449-53.

Ohnishi S, Ohnami S, Laub F, et al (2003). Downregulation and growth inhibitory effect of epithelial-type Kruppel-like transcription factor KLF4, but not KLF5, in bladder cancer. Biochem Biophys Res Commun, 308, 251-6.

Owen GI, Richer JK, Tung L, Takimoto G, Horwitz KB (1998). Progesterone regulates transcription of the p21(WAF1) cyclin- dependent kinase inhibitor gene through Sp1 and CBP/p300. J Biol Chem, 273, 10696-701.

Peiffer L, Poll-Wolbeck SJ, Flamme H, et al (2014). Trichostatin A effectively induces apoptosis in chronic lymphocytic leukemia cells via inhibition of Wnt signaling and histone deacetylation. J Cancer Res Clin Oncol, [Epub ahead of print]

Piacentini P, Conadelli M, Costanzo C, et al (2006). Trichostatin A enhances the response of chemotherapeutic agents in inhibiting pancreatic cancer cell proliferation. Virchows Arch, 448, 797-804.

Platta CS, Greenblatt DY, Kunnimalaiyaan M, Chen H (2007). The HDAC inhibitor trichostatin a inhibits growth of small cell lung cancer cells. J Surg Res, 142, 219-26.

Pourhassan-Moghaddam M, Rahmati-Yamchi M,Akbarzadeh A, et al (2013). Protein detection through different platforms of immuno-loop-mediated isothermal amplification. Nanoscale Res Lett, 8, 485.

Rasheed WK, Johnstone RW, Prince HM (2007). Histone deacetylase inhibitors in cancer therapy. Expert Opin Investig Drugs, 16, 659-78.

Sadat Tabatabaei Mirakabad F, Nejati-Koshki K, Akbarzadeh A, et al (2014). PLGA-based nanoparticles as cancer drug delivery systems. Asian Pac J Cancer Prev, 15, 517-35.

Sawa H, Murakami H, Ohshima Y, et al (2001). Histone deacetylase inhibitors such as sodium butyrate and trichostatin A induce apoptosis through an increase of the bcl-2-related protein Bad. Brain Tumor Pathol, 18, 109-14.

Shan Z, Feng-Nian R, Jie G, Ting Z (2012). Effects of valproic acid on proliferation, apoptosis, angiogenesis and metastasis of ovarian cancer in vitro and in vivo. Asian Pac J Cancer Prev, 13, 3977-82.

Shie JL, Chen ZY, Fu M, Pestell RG, Tseng CC (2000). Gut enriched kruppel-like factor represses cyclin D1 promoter activity through Sp1 motif. Nucleic Acids Res, 28, 2969-76.

Shields JM, Christy RJ, Yang VW (2009). Identification and characterization of a gene encoding a gut-enriched Kruppellike factor expressed during growth arrest. J Biol Chem, 271, 20009-17.

Sonnemann J HI, Sigler M, Palani CD, et al (2008). Histone deacetylase inhibitors and aspirin interact synergistically to induce cell death in ovarian cancer cells. Oncol Rep, 20, 219-24.

Van LC, Verdin E, Verdin E (1996). Expression of a small fraction of cellular genes is changed in response to histone hyperacetylation, Gene Expr, 5, 245-53.

Vigushin DM, Ali S, Pace PE, et al (2001). Trichostatin A is a histone deacetylase inhibitor with potent antitumor activity against breast cancer in vivo. Clin Cancer Res, 7, 971-76.

Wang F, Qi Y, Li X, et al (2013). HDAC inhibitor trichostatin A suppresses esophageal squamous cell carcinoma metastasis through HADC2 reduced MMP-2/9. Clin Invest Med, 36, 87-94.

Wang J, Place RF, Huang V, et al (2010). Prognostic value and function of KLF4 in prostate cancer: RNAa and vector mediated overexpression identify KLF4 as an inhibitor of tumor cell growth and migration. Cancer Res, 70, 10182-91.

Wei D, Gong W, Kanai M, et al (2005). Drastic down-regulation of Kruppel-like factor 4 expression is critical in human gastric cancer development and progression. Cancer Res, $\mathbf{6 5}, 2746-54$.

Wei D, Kanai M, Jia Z, Le X, Xie K (2008). Krüppel-like Factor 4 induces p27Kip1 expression in and suppresses the growth and metastasis of human pancreatic cancer cells. Cancer Res, 68, 4631-9.

Weichert W, Roske A, Gekeler V, et al (2008). Histone deacetylases 1, 2 and 3 are highly expressed in prostate cancer and HDAC2 expression is associated with shorter PSA relapse time after radical prostatectomy. Br J Cancer, 98, 604-10.

Wilson AJ, Holson E, Wagner F, et al (2011). The DNA damage mark pH2AX differentiates the cytotoxic effects of small molecule HDAC inhibitors in ovarian cancer cells. Cancer Biol Ther, 12, 484-93.

Wu ZQ, Zhang R, Chao C, Zhang JF, Zhang YQ (2007). Histone deacetylase inhibitor trichostatin A induced caspaseindependent apoptosis in human gastric cancer cell. Chin Med J, 120, 2112-8.

Yoon HS, Chen X, Yang VW (2003). Kruppel-like factor 4 mediates p53-dependent G1/S cell cycle arrest in response to DNA damage. J Biol Chem, 278, 2101-5.

Yoon HS, Yang VW (2004). Requirement of Kruppel-like factor 4 in preventing entry into mitosis following DNA damage. $J$ Biol Chem, 279, 5035-41.

Yoon O, Roh J (2012). Downregulation of KLF4 and the Bcl-2/ Bax ratio in advanced epithelial ovarian cancer. Oncol Lett, 4, 1033-6.

Yori JL, Seachrist DD, Johnson E, et al (2011). Kruppel-like factor 4 inhibits tumorigenic progression and metastasis in a mouse model of breast cancer. Neoplasia, 13, 601-10.

You BR, Park WH (2013). Trichostatin A induces apoptotic cell death of HeLa cells in a Bcl-2 and oxidative stress-dependent manner. Int J Oncol, 42, 359-66.

Yu F, Li J, Chen H, et al (2011). Kruppel-like factor 4 (KLF4) is required for maintenance of breast cancer stem cells and for cell migration and invasion. Oncogene, 30, 2161-72.

Zhang QC, Jiang SJ, Zhang S, Ma XB (2012). Histone deacetylase inhibitor trichostatin A enhances anti-tumor effects of docetaxel or erlotinib in A549 Cell Line. Asian Pac J Cancer Prev, 13, 3471-6.

Zhang S, Zhang QC, Jiang SJ, Chin SJ (2013). Effect of trichostatin A and paclitaxel on the proliferation and apoptosis of lung adenocarcinoma cells. Chin Med J, 126, 129-34.

Zhang W, Geiman DE, Shields JM (2000). The gut enriched Kruppel-like factor (Kruppel-like factor 4) mediates the transactivating effect of p53 on the p21WAF1/Cip1 promoter. J Biol Chem, 275, 18391-8. 